Original Article

\title{
A simple method for estimating the intervertebral disc compressive force based on the posture analysis of community-dwelling older adults
}

\author{
Azusa Nishizawa, PO1)*, Junji Katsuhira, PhD $^{2)}$, Makoto Watanabe, RPT ${ }^{1}$, \\ Hiroyuki OKa, MD, PhD ${ }^{3)}$, Ko Matsudaira, MD, PhD ${ }^{3)}$ \\ 1) Prosthetics \& Orthotics and Assistive Technology, Niigata University of Health and Welfare: 1398 \\ Shimamicho, Kita-ku, Niigata-shi, Niigata 950-3198, Japan \\ 2) Department of Human Environment Design, Faculty of Human Life Design, Toyo University, Japan \\ 3) Department of Medical Research and Management for Musculoskeletal Pain, 22nd Century Medical \\ and Research Center, Faculty of Medicine, The University of Tokyo Hospital, Japan
}

\begin{abstract}
Purpose] The purpose of this study was to develop a simpler method to estimate the intervertebral disc compressive force in healthy older adults. We also examined the validity of a simpler estimation formula for patients with spinal diseases. [Participants and Methods] Fifty-two older adults participated in the study. The standing posture was measured using a three-dimensional motion capture system. The intervertebral disc compressive force was calculated using a previously reported method. Correlation analysis was used to detect the relationship between the measured parameters and the intervertebral disc compressive force. Multiple regression analysis was performed to obtain an equation for the intervertebral disc compressive force. Correlation analysis was used to determine the regression equation for the patients with spinal diseases. [Results] Multiple regression analysis showed that trunk flexion/extension angle and body mass were significantly associated with intervertebral disc compressive force. A correlation was found between the measured and predicted values in the healthy older adults, whereas both values were inconsistent in patients with spinal diseases. [Conclusion] The results of our study demonstrated that the trunk flexion/extension angle and body mass are indicators of intervertebral disc compressive force and can be used to assess low back mechanical stress in healthy older adults.

Key words: Low back mechanical stress, Motion analysis, Elderly
\end{abstract}

(This article was submitted Jan. 5, 2021, and was accepted Feb. 23, 2021)

\section{INTRODUCTION}

Low back pain (LBP) is a major problem globally, with $85 \%$ of all people experiencing it in their lifetime; LBP also worsens the quality of life for the elderly ${ }^{1,2)}$. LBP is generally caused by ergonomics and psychosocial stress, among other reasons $^{3)}$. Smith et al. ${ }^{4)}$ reported that postural changes that deviate from the neutral posture were associated with a high risk of LBP development.

The ability to make postural adjustments gradually declines with age, resulting in a misalignment of the trunk and the lower extremities ${ }^{5,6}$. Previous studies have reported that age-related changes in the posture as well as the pelvic backward tilt and anterior deviation of the center of gravity (COG) are typical postures of the Japanese elderly ${ }^{7-9)}$.

Hasegawa et al. ${ }^{10)}$ used the intervertebral disc compressive force estimated from the low back moments as an index of low back mechanical stress and demonstrated that participants with LBP have higher intervertebral disc compressive force than those without LBP. The intervertebral disc compressive force is affected because of postural changes, and a continuous high

*Corresponding author. Azusa Nishizawa (E-mail: ham19003@nuhw.ac.jp)

(C2021 The Society of Physical Therapy Science. Published by IPEC Inc.

(c) (i) $\odot$ This is an open-access article distributed under the terms of the Creative Commons Attribution Non-Commercial No DerivaCC BY NC ND tives (by-nc-nd) License. (CC-BY-NC-ND 4.0: https://creativecommons.org/licenses/by-nc-nd/4.0/) 
stress on the intervertebral disc would lead to LBP.

However, few studies have reported an association between the intervertebral disc compressive force and posture in the elderly. Considering the large population of the elderly and their aging process, it is crucial to understand the factors that influence the intervertebral disc compressive force in the elderly. The purpose of this study is two-fold: 1) after evaluating the intervertebral disc compressive force of the elderly, we sought a simpler method to estimate the intervertebral disc compressive force of the elderly without complaints of LBP; 2) we also examined the validity of the simpler estimation formula for elderly people with LBP.

\section{PARTICIPANTS AND METHODS}

The participants were 52 community-dwelling elderly individuals, aged older than 65 years. The study protocol was approved by the ethics committee of Niigata University of Health and Welfare (approved numbers: 18336-191220). Informed consent was obtained from all participants before the study.

The participants completed a self-reported questionnaire including age, body height, body mass, medical history, and the Roland-Morris Disability Questionnaire (RDQ) prior to measurement. The RDQ was used to separate participants with or without LBP ${ }^{11,12)}$. Based on the self-reported questionnaire, the participants were divided into two groups-healthy group (HG) and spinal disease group (SG). HG included participants having no medical history in the last two years and without LBP while participating in the experiment. SG included members who had been diagnosed with and received treatment for orthopedic diseases of the spine region or who had an RDQ score higher than 1, indicating the presence of LBP that interferes with daily life.

The experimental task was to stand statically. A three-dimensional (3D) motion capture system with 12 infrared cameras (VICON MX; Vicon Motion Systems Ltd., Oxford, UK) and two force plates (OR6-6-2000; AMTI, MA, USA) was used to record marker coordinates and floor reaction force data. Participants were instructed to look straight ahead and stand with their feet apart (to match the width between the shoulders) and placed on separate force plates. The posture was measured for $10 \mathrm{~s}$ using the $3 \mathrm{D}$ motion capture system. The measurement was repeated thrice with an interval allowed between the trials.

The sampling frequencies of the infrared cameras and the force plates were 100 and 1,000 $\mathrm{Hz}$, respectively. Infrared reflective markers were attached to the participants' bodies at the parietal, occipital ridges, glabella, 7th cervical vertebra, 10th thoracic vertebra, midway between the 4th and 5th lumbar vertebra, sacrum, anterior superior iliac spine, posterior superior iliac spine, acromion process, lateral epicondyle of the humerus, radial styloid process, hip joints, front of the thigh, medial epicondyle of the femur, lateral epicondyle of the femur, tibial tuberosities, medial malleolus, lateral malleolus, and 2nd metatarsophalangeal joint. In addition, two cluster markers were placed on the 10th thoracic vertebrae for the kinematic calculation of spinal alignment. In total, thirty-seven infrared reflective markers were attached to the participants, as indicated in Fig. 1.

The analysis program was developed using Visual 3D version 6 (C-motion, Inc., Germantown, MD, USA). The marker coordinates and the floor reaction force data were processed through low pass filtering at 6 and $15 \mathrm{~Hz}$, respectively, using Winter's method ${ }^{13)}$. For the kinematic evaluation of the posture, the trunk comprised the 10th thoracic vertebrae and the cluster markers, and the pelvis comprised the left and right posterior superior iliac spines and the sacrum. In this study, the pelvis angle, trunk angle, and trunk angle relative to the pelvis were used as indices for the posture. The angles of the pelvis and trunk were angles of each segment relative to the global coordinate system, and the trunk angle relative to the pelvis was the relative angle between each segment.

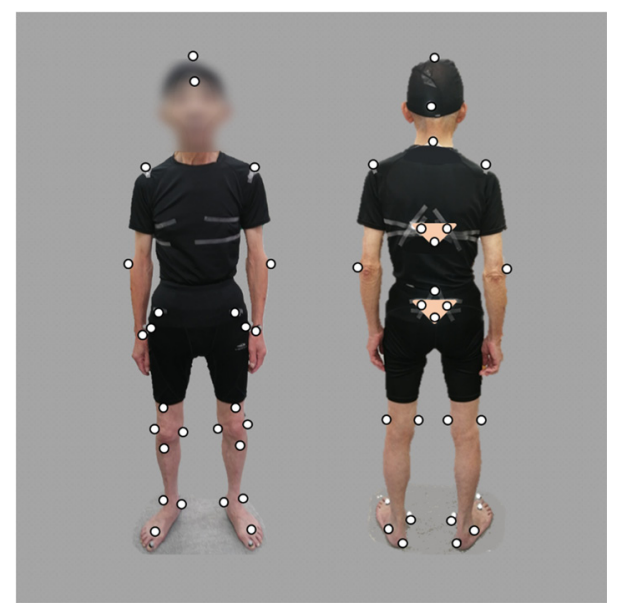

Fig. 1. Positions of infrared reflective markers on the participant's body. 
The intervertebral disc compressive force was used as the indicator of low back mechanical stress. This calculation method was reported previously ${ }^{10,14)}$ (Eq. 1).

Intervertebral disc compressive force $(\mathrm{N}) \approx$

$+20 \mid$ Extension moment $\mid$ or 13 | Flexion moment $\mid$

$+8 \mid$ Side bending moment $\mid$

$+23 \mid$ Rotation moment

+ Gravitational force acting on Center of gravity of HAT $\cdot \cos \theta$ Eq. 1

where $\theta$ is the two-dimensional angle between the trunk and the vertical line in the sagittal plane, and HAT indicates "Head, Arms, and Trunk." Each coefficient represents the moment arm from the center of the intervertebral disc to an individual muscle. Specifically, the coefficient is equal to the distance between the intervertebral disc and the paraspinal erector muscle when the lumbar extension moment occurs, or the distance between the intervertebral disc and the rectus abdominis muscle when the lumbar flexion moment occurs.

All parameters are shown as the average values of three trials. Low back moments were normalized by the participants' body mass. Absolute values were used for the angles and moments of the coronal and the horizontal plane to avoid being canceled because of changes in the opposite direction. The data were checked for outliers using the Smirnov-Grubbs test, and any participants having rejected values were excluded from the subsequent analysis. Pearson's correlation coefficient was used to investigate the correlation with intervertebral disc compressive forces, physical information such as body mass and body height, low back moment, and posture parameters in HG. To investigate factors affecting the intervertebral disc compressive force, a stepwise multiple regression analysis was applied. Parameters exhibiting moderate or higher correlations $(|r|>0.4)$ with the intervertebral disc compressive force were selected as independent variables. Finally, the obtained multiple regression equation was applied to SG, and those predicted values were evaluated using the Pearson's correlation coefficient. The coefficients with $\mathrm{p}<0.05$ were considered significant. SPSS software version 24.0 (IBM, IL, USA) was used for the statistical analysis.

\section{RESULTS}

Thirty-seven elderly were included in HG and fifteen elderly were included in SG. One HG participant was excluded on account of being considered an outlier by the Smirnov-Grubbs test. Therefore, the final count was 36 and 15 participants in HG and SG, respectively (Table 1). The intervertebral disc compressive force of HG was $512.68 \pm 108.38 \mathrm{~N}$ (mean $\pm \mathrm{SD}$ ). The results of a correlation analysis of intervertebral disc compressive force and anthropometry parameters are shown in Table 2. Body mass and BMI have a significant correlation with intervertebral disc compressive force $\left(\mathrm{R}^{2}=0.49\right.$ and 0.37 , $\mathrm{p}<0.01$ and $<0.01$, respectively). Table 3 lists the correlation between the intervertebral disc compressive force and posture parameters. A significant correlation was observed between the trunk flexion/extension angle and the intervertebral disc compressive force $\left(\mathrm{R}^{2}=0.16, \mathrm{p}=0.02\right)$.

Multiple regression analysis was calculated to predict the intervertebral disc compressive force based on trunk flexion/ extension angle and body mass, as indicated in Table 4. Significant regression was observed $(F(2,33)=21.31)$, $p<0.001)$. The predicted intervertebral disc compressive force increased to $8.33 \mathrm{~N}$ for each kilogram of body mass and decreased to 4.58 $\mathrm{N}$ for when the trunk extended by $1^{\circ}$. Multicollinearity $(|\mathrm{r}|>0.7)$ did not exist between the independent variables, and the Durbin-Watson ratio was 1.82 .

Figure 2 shows the scatter plots of the measured and predicted intervertebral disc compressive forces for the two groups. In contrast to the result of $\mathrm{HG}, \mathrm{SG}$ had a coefficient of determination of 0.0004 , and the predicted values from the multiple regression equation were different from the measured value obtained using Eq. 1.

\section{DISCUSSION}

We measured the posture of the pelvis and the trunk in the elderly using a 3D motion capture system and investigated

Table 1. Anthropometry characteristics of each group

\begin{tabular}{lcc}
\hline & Healthy group & $\begin{array}{c}\text { Spinal disease } \\
\text { group }\end{array}$ \\
\hline $\mathrm{n}$ & 36 & 15 \\
Gender (\% of male) & $61 \%$ & $67 \%$ \\
Age (SD), years & $71.7(4.1)$ & $71.0(3.3)$ \\
Body height (SD), cm & $159.8(8.3)$ & $160.9(7.3)$ \\
Body mass (SD), kg & $58.4(8.5)$ & $58.8(8.1)$ \\
BMI (SD), $\mathrm{kg} / \mathrm{m}^{2}$ & $22.8(2.4)$ & $22.7(2.4)$ \\
\hline
\end{tabular}

SD: standard deviation.
Table 2. Results of correlation analysis between intervertebral disc compressive force and self-reported questionnaire in healthy group

\begin{tabular}{lcc}
\hline & $\mathrm{R}^{2}$ & $\mathrm{p}$ \\
\hline Age & 0.04 & 0.23 \\
Body height & 0.12 & $0.04^{*}$ \\
Body mass & 0.49 & $<0.01^{*}$ \\
BMI & 0.37 & $<0.01^{*}$ \\
\hline $\mathrm{R}^{2}$ is the determination coefficient; *Significant \\
difference between the conditions $(\mathrm{p}<0.05)$.
\end{tabular}


Table 3. Results of correlation analysis between intervertebral disc compressive force and posture parameters in healthy group

\begin{tabular}{llcc}
\hline & & $\mathrm{R}^{2}$ & $\mathrm{p}$ \\
\hline \multirow{2}{*}{ Pelvis } & Tilt & 0.00 & 0.76 \\
& Lateral bending & 0.00 & 0.84 \\
Trunk & Flexion/extension & 0.16 & $0.02^{*}$ \\
& Lateral bending & 0.02 & 0.38 \\
\multirow{3}{*}{ Trunk relative to pelvis } & Flexion/extension & 0.09 & 0.08 \\
& Lateral bending & 0.00 & 0.91 \\
& Rotation & 0.06 & 0.14 \\
\hline
\end{tabular}

$\mathrm{R}^{2}$ is the determination coefficient; *Significant difference between the conditions $(\mathrm{p}<0.05)$

Table 4. Results of multiple regression analysis

\begin{tabular}{lrrrc}
\hline & $\mathrm{B}$ & $\beta$ & $\mathrm{p}$ & \multicolumn{1}{c}{$95 \%$ CI } \\
\hline Constant & 66.13 & & 0.49 & -124.58 to 256.83 \\
Body mass & 8.33 & 0.65 & $<0.01$ & 5.27 to 11.40 \\
Trunk F/E angle & -4.58 & -0.28 & 0.02 & -8.53 to -0.63 \\
\hline
\end{tabular}

$\mathrm{B}$ is the partial regression coefficient; $\beta$ is the standardized partial regression coefficient; $95 \% \mathrm{CI}$ : $95 \%$ confidence interval; F/E: flexion/extension; $\mathrm{R}^{2}$ : determination coefficient. $\mathrm{R}^{2}=0.56$, ANOVA $\mathrm{p}<0.001$.

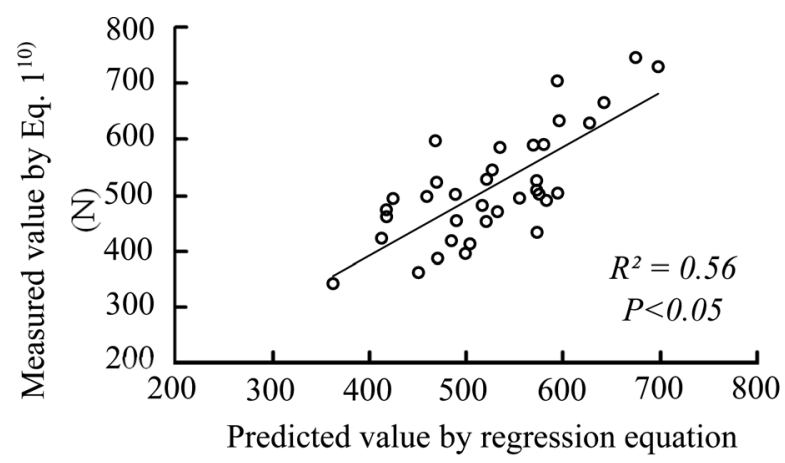

$(\mathrm{N})$

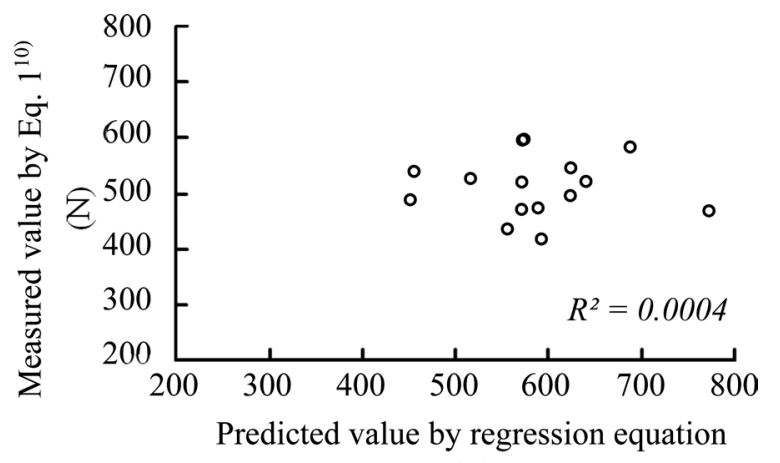

$(\mathrm{N})$

Fig. 2. Scatter plots of the intervertebral disc compressive force calculated by Eq. 1 and predicted by the multiple regression equation for the healthy (left) and spinal disease (right) groups. Eq. 1 is referenced in "PARTICIPANTS AND METHODS".

the effect of posture on the low back mechanical stress of the elderly. The intervertebral disc compressive force used as an indicator of low back mechanical stress herein was the estimated value calculated from the low back moments. Previous studies that measured the disc compression force by inserting a pressure gauge directly into the volunteer's intervertebral disc reported intervertebral disc compressive force of $0.27-0.54 \mathrm{MPa}$ during the upright posture ${ }^{15-17)}$. These are equivalent to $486-972 \mathrm{~N}$ when converted into forces based on a $1,800 \mathrm{~mm}^{2}$ cross-sectional area of the intervertebral disc reported by Wilke et $\mathrm{al}^{15)}$. Furthermore, the pressure applied to the intervertebral disc in the standing position was 444 to $594 \mathrm{~N}^{18)}$. Therefore, the average intervertebral disc compressive force herein was nearly within the range reported by previous studies; this confirmed that our study did not overestimate or underestimate the force.

As a result of the correlation and multiple regression analyses, the body mass and trunk flexion/extension angle are related to intervertebral disc compressive force. As the spinal load includes the gravity applied to the HAT and body mass influence the spinal load ${ }^{19)}$. Among the posture parameters, the trunk flexion/extension angle had a considerable effect on the intervertebral disc compressive force. The low back moments were affected by the moment arm and ground reaction force. The moment arm is defined as the distance between the COG of the HAT and the joint center. Previous studies have reported the importance of the COG shifts on the sagittal plane, stating that sagittal alignment changes could prompt LBP and prolong symptoms ${ }^{15,16,20)}$. Furthermore, the anterior deviation of the COG with trunk flexion is a typical posture of the Japanese elderly ${ }^{9}$. Therefore, an increase in the trunk flexion extends the moment arm and consequently increases demands on the 
low back extension moment to maintain posture. This increased moment affects the intervertebral disc compressive force.

The regression equation obtained for predicting the intervertebral disc compressive force was evaluated by comparing both measured and predicted values of intervertebral disc compressive force. The measured and predicted values of intervertebral disc compressive force were in good agreement in HG, whereas this was not the case in SG. This result could be due to specific posture of participants in SG. Patients with spinal diseases or those experiencing LBP are known to assume a specialized posture due to their condition. Previous studies have reported an increase in the sagittal vertical axis and altered pelvic tilt angle in patients with spinal disease and $\mathrm{LBP}^{21},{ }^{22}$. In this study, there was a difference in the pelvic tilt angles of the two groups ( $\mathrm{HG}, 16.19 \pm 6.17^{\circ} ; \mathrm{SG}, 1.88 \pm 3.59^{\circ} ; \mathrm{p}<0.01$ ). It is possible that the difference in the pelvis causes a compensatory posture of the trunk and other joints and affects the low back load.

The results of this study suggest that the trunk flexion/extension angle and body mass affect lumbar mechanical stress in the elderly. Furthermore, the regression equation of the intervertebral disc compressive force estimates the intervertebral disc compressive force simply by measuring the inclination angle of the trunk and body mass. Therefore, the regression equation is useful for the evaluation and rehabilitation of diseases caused by mechanical load, including LBP.

Our study has some limitations. First, the participants of this study were recruited from one community, and their body mass and height were self-reported. Further, we investigated only the standing posture; therefore, investigating other activities, such as gait, is necessary. Finally, the intervertebral disc compressive force was determined by the inverse dynamic estimation method. Thus, co-contraction between the muscles as well as the physiological changes of the intervertebral disc was not considered. However, a previous study stated that neglecting co-contraction between agonist and antagonist muscles was trivial in spinal load estimation ${ }^{23}$. Further studies should involve an increased number of participants. In a future study, the authors also intend to focus on other activities of daily living, such as walking, and postural intervention decreasing the intervertebral disc compressive force.

\section{Funding}

This study was supported by a research grant from the Japanese Society of Musculoskeletal Medicine.

\section{Conflict of interest}

JK is a shareholder (unlisted) and a director of Trunk Solution CO., Ltd. and received the following support: a research grant from JSPS KAKENHI.

HO received grants from Teijin Pharma Limited, Pfizer Inc., and Fujifilm Medical Co., Ltd. and grants and personal fees from Nippon Zoki Pharmaceutical Co., Ltd., Ono Pharmaceutical Co., Ltd., Chugai Pharmaceutical Co., Ltd., AYUMI Pharmaceutical Corporation, Sompo Holdings, Inc., MS\&AD InterRisk Research \& Consulting, Inc., Inotech Co., Ltd., NUVASIVE Japan, Medical Data Scientist, and Medical AI Device Development Organization, The Association for Preventive Medicine of Japan, MTG Co., Ltd., and Shionogi \& Co., Ltd. apart from that for the submitted work.

KM is a shareholder and an adviser of Trunk Solution CO., Ltd. He submitted work and received a research grant from the Ministry of Health, Labour and Welfare, grant support from Sumitomo Dainippon Pharma Co., Ltd. and Okamura Corporation, and grant support, including personal fees, from Nippon Zoki Pharmaceutical Co., Ltd., Ono Pharmaceutical Co., Ltd., Chugai Pharmaceutical Co., Ltd., AYUMI Pharmaceutical Corporation, Sompo Holdings, Inc., MS\&AD InterRisk Research \& Consulting, Inc., Inotech Co., Ltd., NUVASIVE Japan, Medical Data Scientist and Medical AI Device Development Organization, The Association for Preventive Medicine of Japan, MTG Co., Ltd., and Shionogi \& Co., Ltd. He also received lecture fees from Eli Lilly Japan K.K., Astellas Pharma Inc., TOTO Ltd., Eisai Co., Ltd., Pfizer Japan Inc., Hisamitsu Pharmaceutical Co., Inc., Janssen Pharmaceutical K.K., Kaken Pharmaceutical Co., Ltd., and Teijin Pharma Limited. Furthermore, he received lecture and advisory fees from Murata Manufacturing Co., Ltd. outside the submitted work.

\section{REFERENCES}

1) Fujii T, Matsudaira K: Prevalence of low back pain and factors associated with chronic disabling back pain in Japan. Eur Spine J, 2013, 22: 432-438. [Medline] [CrossRef]

2) Airaksinen O, Brox JI, Cedraschi C, et al. COST B13 Working Group on Guidelines for Chronic Low Back Pain: Chapter 4. European guidelines for the management of chronic nonspecific low back pain. Eur Spine J, 2006, 15: S192-S300. [Medline] [CrossRef]

3) Matsudaira K, Konishi H, Miyoshi K, et al.: Potential risk factors for new onset of back pain disability in Japanese workers: findings from the Japan epidemiological research of occupation-related back pain study. Spine, 2012, 37: 1324-1333. [Medline] [CrossRef]

4) Smith A, O'Sullivan P, Straker L: Classification of sagittal thoraco-lumbo-pelvic alignment of the adolescent spine in standing and its relationship to low back pain. Spine, 2008, 33: 2101-2107. [Medline] [CrossRef]

5) Gelb DE, Lenke LG, Bridwell KH, et al.: An analysis of sagittal spinal alignment in 100 asymptomatic middle and older aged volunteers. Spine, 1995, 20: 1351-1358. [Medline] [CrossRef]

6) Gong H, Sun L, Yang R, et al.: Changes of upright body posture in the sagittal plane of men and women occurring with aging - a cross sectional study. BMC Geriatr, 2019, 19: 71. [Medline] [CrossRef]

7) Yukawa Y, Kato F, Suda K, et al.: Normative data for parameters of sagittal spinal alignment in healthy subjects: an analysis of gender specific differences and changes with aging in 626 asymptomatic individuals. Eur Spine J, 2018, 27: 426-432. [Medline] [CrossRef] 
8) Hasegawa K, Okamoto M, Hatsushikano S, et al.: Normative values of spino-pelvic sagittal alignment, balance, age, and health-related quality of life in a cohort of healthy adult subjects. Eur Spine J, 2016, 25: 3675-3686. [Medline] [CrossRef]

9) Taniguchi N, Ijiri K, Matsunaga S, et al.: Radiographic analysis of sagittal spinal alignment and balance in eldery individuals. Orthop Trauma, 2000, 49: 682-684 (in Japanese). [CrossRef]

10) Hasegawa T, Katsuhira J, Oka H, et al.: Association of low back load with low back pain during static standing. PLoS One, 2018, 13: e0208877. [Medline] [CrossRef]

11) Roland M, Morris R: A study of the natural history of back pain. Part I: development of a reliable and sensitive measure of disability in low-back pain. Spine, 1983, 8: 141-144. [Medline] [CrossRef]

12) Suzukamo Y, Fukuhara S, Kikuchi S, et al. Committee on Science Project, Japanese Orthopaedic Association: Validation of the Japanese version of the Roland-Morris disability questionnaire. J Orthop Sci, 2003, 8: 543-548. [Medline] [CrossRef]

13) Winter DA: Biomechanics and Motor control of Human Movement, 3rd ed. Hoboken: Wiley, 2004.

14) Katsuhira J, Matsudaira K, Iwakiri K, et al.: Effect of mental processing on low back load while lifting an object. Spine, 2013, 38: E832-E839. [Medline] [CrossRef]

15) Wilke HJ, Neef P, Caimi M, et al.: New in vivo measurements of pressures in the intervertebral disc in daily life. Spine, 1999, 24: 755-762. [Medline] [CrossRef]

16) Sato K, Kikuchi S, Yonezawa T: In vivo intradiscal pressure measurement in healthy individuals and in patients with ongoing back problems. Spine, 1999,24 : 2468-2474. [Medline] [CrossRef]

17) Schultz A, Andersson G, Ortengren R, et al.: Loads on the lumbar spine. Validation of a biomechanical analysis by measurements of intradiscal pressures and myoelectric signals. J Bone Joint Surg Am, 1982, 64: 713-720. [Medline] [CrossRef]

18) Dreischarf M, Shirazi-Adl A, Arjmand N, et al.: Estimation of loads on human lumbar spine: a review of in vivo and computational model studies. J Biomech, 2016, 49: 833-845. [Medline] [CrossRef]

19) Ghezelbash F, Shirazi-Adl A, Arjmand N, et al.: Effects of sex, age, body height and body weight on spinal loads: sensitivity analyses in a subject-specific trunk musculoskeletal model. J Biomech, 2016, 49: 3492-3501. [Medline] [CrossRef]

20) Chun SW, Lim CY, Kim K, et al.: The relationships between low back pain and lumbar lordosis: a systematic review and meta-analysis. Spine J, 2017, 17: 1180-1191. [Medline] [CrossRef]

21) Suzuki H, Endo K, Kobayashi H, et al.: Total sagittal spinal alignment in patients with lumbar canal stenosis accompanied by intermittent claudication. Spine, 2010, 35: E344-E346. [Medline] [CrossRef]

22) Jackson RP, McManus AC: Radiographic analysis of sagittal plane alignment and balance in standing volunteers and patients with low back pain matched for age, sex, and size. A prospective controlled clinical study. Spine, 1994, 19: 1611-1618. [Medline] [CrossRef]

23) van Dieën JH, Kingma I: Effects of antagonistic co-contraction on differences between electromyography based and optimization based estimates of spinal forces. Ergonomics, 2005, 48: 411-426. [Medline] [CrossRef] 